\title{
Eversion technique: A safe anastomosis method of bile duct in living donor liver transplantation without internal or external biliary stent
}

\author{
Seok-Hwan KIM
}

Department of Surgery, Chungnam National University Hospital, Daejeon, Korea

Introduction: Biliary stricture (BS) is still a major concern after bile duct anastomosis in living donor liver transplantation (LDLT), even after the technical refinements using a microscope. This study aims to describe our eversion technique without stent insertion of biliary anastomosis and its effects on the incidence of biliary complications.

Methods: This was a single-center retrospective study of 52 adult LDLT recipients between December 2011 and June 2020. Group 1 consisted of the first 20 patients for whom the standard technique of biliary anastomosis (minimal hilar dissection during donor duct division, high hilar division of the recipient bile duct, and preservation of the recipient duct periductal tissue) was used. Group 2 consisted of 32 patients for whom biliary anastomosis was done with the addition of corner-sparing sutures and mucosal eversion of the recipient duct to the standard technique. Primary outcome measures included biliary complications (biliary leaks and strictures).

Results: Biliary complications occurred in 4/20 patients in group $1(20.0 \%)$ and 2/32 patients in group 2 (6.25\%). The technical factors mentioned above are aimed at preserving the blood supply of the donor and recipient ducts and hold the key for minimizing biliary complications in adult-to-adult LDLT.

Conclusions: The application of eversion technique could be a key for preventing BAS in duct-to-duct biliary reconstruction in LDLT. 